\title{
Morfometria de Papilioninae (Lepidoptera, Papilionidae) ocorrentes em quatro localidades do Rio Grande do Sul, Brasil. III. Análise da forma das asas através de marcos anatômicos
}

\author{
Rocco Alfredo Di Mare ${ }^{1} \&$ Elio Corseuil ${ }^{2}$ \\ ${ }^{1}$ Departamento de Biologia, Centro de Ciências Naturais e Exatas, Universidade Federal de Santa Maria. Campus \\ Universitário, Faixa de Camobi, km 9, 97115-900 Santa Maria, Rio Grande do Sul, Brasil. E-mail: ram13@ terra.com.br. \\ ${ }^{2}$ Curso de Pós-Graduação em Biociências, Pontifícia Universidade Católica do Rio Grande do Sul. Caixa Postal 1429, \\ 90619-900 Porto Alegre, Brasil. E-mail: corseuil@pucrs.br
}

\begin{abstract}
Morphometrics of Papilioninae (Lepidoptera, Papilionidae) occurring in four communities from Rio Grande do Sul, Brazil. III. Shape wing analysis by landmarks. This study investigated variation in the forewing morphology of 11 butterfly species (Papilioninae), sampled in four communities of the Rio Grande do Sul State. The analyses were performed using descriptors of shape derived from anatomical landmarks, wing areas, and area, length and width of the discal cell. Differences in the consensus configuration for wing shape between male and female wings were not significant, showing a high correlation degree. The wing shape did not differ among the four communities investigated. The observed differences in wing shape of the species studied here should be better explained by independent changes associated with different areas of the wing, mainly with the discal cell.
\end{abstract}

KEY WORDS. Consensus configuration, landmarks, partial warps, shape components.

RESUMO. Neste estudo investigou-se a variação na morfologia das asas anteriores de 11 espécie de Papilioninae, coletadas em quatro localidades do Rio Grande do Sul. As análises foram realizadas usando descritores de forma a partir de marcos anatômicos, área das asas, e área, comprimento e largura da célula discal. Diferenças na configuração de consenso para a forma da asa entre as asas dos machos e das fêmeas não foram significantes, embora apresentem um grau de correlação elevado. A forma da asa não diferiu entre as quatro comunidades investigadas. As diferenças observadas na forma de asa das espécies estudadas poderiam ser mais bem explicadas por mudanças independentes associadas com diferentes áreas da asa, principalmente com a célula discal.

PALAVRAS CHAVE. Componente uniforme, configuração de consenso, marcos anatômicos, deformação relativa.

Embora a análise da forma possa ser investigada através de métodos matemáticos tradicionais (MARCus 1990), nota-se um interesse renovado na comparação direta da forma dos organismos (Bookstein 1991, Marcus 1993, Rohlf 1993). Entre estes métodos diretos utilizados se destaca a utilização de marcos anatômicos, para representar as formas de uma maneira compreensiva através de dados numéricos médios. Os dados podem ser obtidos através de coordenadas médias de marcos distribuídos perifericamente, ou dentro das estruturas analisadas, ou das distâncias entre os marcos anatômicos escolhidos diretamente para cada espécie.

Nos lepidópteros, a morfologia das asas incluindo características do tamanho, da forma, pigmentação e variação fenotípica têm desempenhado papel importante em estudos taxonômicos e evolutivos, embora os aspectos quantitativos de forma das asas, também, sejam empregados às vezes em es- tudos de padrões fenotípicos (MASOn et al. 1968, BRAKEFIELD 1979, Kingsolver \& Wiernasz 1987), dimorfismo sexual (Pearse \& Murray 1982) e comportamento de vôo (Nachtigall 1967, Alexander 1983, Corkum 1987). Entretanto, limitações funcionais e arquitetônicas podem refletir padrões conservativos e estáveis de covariação, entre caracteres que são normalmente negligenciados em estudos de evolução morfológica (STRAuss 1990).

As estratégias de comportamento adotadas por um indivíduo podem depender diretamente do seu fenótipo e no modo que ele interage com o ambiente. Nos organismos que voam, padrões de vôo distintos podem necessitar condições fisiológicas e bioquímicas diferentes (DudLEy 2000). A forma e o tamanho do corpo devem ser vistos como resultado de um compromisso entre a reprodução máxima imediata e o investimento de energia assimilada para crescer, sobreviver e se reproduzir (Harder 1985, Barbault 1988). Alterações na adaptabilidade re- 
sultam de pressões ecológicas relacionadas com energia, termorregulação, predação, competição intra e interespecífica, entre outros, determinando os coeficientes de sel eção que usam o tamanho e a forma do corpo como uma característica central.

Segundo BARBAULt (1988), o tamanho e a forma têm sido usualmente analisados pelos ecologistas simplesmente como variáveis distintivas, em vez de uma característica central dependente de características fisiológicas, ecológicas e demográficas. Há uma desaten ção para com a importância do tamanho e a forma que os organismos apresentam em relação às restrições ecológicas e pressões sel etivas potenciais, tais como: competição intra-específica, predação, parasitismo e condições metabólicas que podem exercer influências sobre estas características. Normalmente, a associação é com a competição intraespecífica. Portanto, dados sobre a diversidade morfológica são importantes, tanto nos estudos ecológicos quanto evolutivos (RoHLf 1990).

Em Papilionidae, a subfamília Papilioninae é a maior, subdividida em três tribos: Leptocircini Kirby, 1896 [=Graphiini Talbot, 1939], Papilionini e Troidini. No Brasil, existem cerca de 73 espécies registradas para esta subfamília, distribuídas em nove gêneros: Battus Scopoli, 1777, Euryades Felder \& Felder, 1864, Eurytides Hübner, 1821, Heraclides Hbn., 1819, M imoides K. Brown, 1991, Parides Hübner, 1819, Protesilaus Swainson, 1832, Protographium e Pterourus Scopoli, 1777 (TyLer et al. 1994). TESTON \& CORSEUIL (1998) listaram para o Rio Grande do Sul 37 representantes da família Papilionidae, incluindo espécies e subespécies. Na revisão bibliográfica realizada, não foram encontrados estudos que analisam quantitativamente a morfologia de Papilioninae no Brasil. Os estudos estão relacionados, principalmente, com a biologia, taxonomia, demografia, mimetismo, ecologia e reprodução de Papilionidae (D'ALMEIDA 1966, Brown JR. et al. 1981, Morais \& Brown JR. 1992, Teston \& CORSEUIL 1998, entre outros).

Este trabalho tem como objetivo investigar padrões de variação na forma das asas anteriores de Papilioninae, capturados em quatro local idades da Depressão Central do Rio Grande do Sul, através dos padrões obtidos com marcos anatômicos.

\section{MATERIAL E MÉTODOS}

Foram analisadas 520 borboletas (379 machos e 141 fêmeas) pertencentes a 11 espécies de Papilioninae, uma das quais com três subespécies (Tab. I), col etadas em quatro sítios (VDRC =Vale do Diabo + Rincão do Canto; RSML = Rincão do Soturno + Morro do Link; AN = Água Negra; SCCB = Schöenstatt + Camobi) localizados na Depressão Central do Rio Grande do Sul, entre os paral elos 5310' e 5440'W eentre 2900' e 2920'S. As coletas foram realizadas entre setembro de 1994 a março de 1995 e entre novembro de 1995 a fevereiro de 1996, em interval os médios de trinta dias para cada localidade estudada, utilizando rede entomológica convencional, durante períodos de quatro horas. Os adultos coletados foram acondicionados em envelopes de papel vegetal, individualizados, devidamente etiquetados e transportados para o Laboratório de Biologia Evolutiva do Departamento de Biologia da Universidade Federal de Santa Maria (UFSM). A identificação das espécies foi realizada com base em TYLer et al. (1994).

As localidades VDRC, RSML e AN estão localizadas em áreas remanescentes da floresta decidual e semidecidual da Serra Geral, todas em altitudes inferiores a 500 metros. Nelas podem ser distinguidos cinco extratos vegetais: emergente, arbóreo contínuo, arvoretas, arbustivo e herbáceo (KLEIN 1983). Em SCCB, a flora, eminentemente urbana, é constituída de plantas ornamentais cultivadas: Apocynaceae, Asteraceae, Malvaceae, Myrtaceae e Verbenaceae. As localidades podem ser distinguidas pelo percentual da cobertura vegetal, cerca de $70 \%$ em VDRC, $50 \%$ em RSML e 30\% em AN. Água Negra se distingue por sua fisionomia particular: hábitat fragmentado, formado por capões com vegetação lenhosa baixa e de média altura, com abundância de Myrtaceae e Rubiaceae. Em SCCB a cobertura vegetal pode ser considerada mínima, quase inexistente, associada a uma vegetação antrópica (ScHWARTZ \& Dı M ARE 2001).

As asas foram separadas do corpo e fixadas entre folhas de material plástico auto-adesivo transparente, com dimensões aproximadas de $10 \mathrm{~cm} \times 10 \mathrm{~cm}$. Foram escolhidos 22 marcos anatômicos para análise de coordenadas da célula discal (CD) (Fig. 1) e das regiões alares (Fig. 2): área basal (AB) área mediana (AM) e área apical (AP). De acordo com a classificação de BooKSTEIN (1991), os marcos anatômicos estabelecidos no presente trabal ho são do tipo I, exceto o marco 22, que foi obtido através da projeção da reta formada pelos marcos 2 e 16 até a borda basal da asa. As coordenadas dos marcos anatômicos foram digitalizadas com o programa TPSDIG32 (RoHLF 2001). Os val ores das áreas, comprimento elargura da célula discal foram cal culados com o programa UTHSCSA (WILcox et al. 1996). Para que os dados fossem uniformizados, os valores absolutos obtidosforam convertidos em valores percentuais. A proporção das regiões alares foi calculada com base no quociente entre cada área $(C D, A B, A M, A A)$ e a área total $(A B+A M+A A)$. O comprimento relativo da célula discal foi estimado com base no quociente entre as retas formadas pel os pontos 1 e 4 (a) e 4 e 5 (b) $(\mathrm{a} /(\mathrm{a}+\mathrm{b}))($ Fig. 3), e a largura entre as retas formadas pelos pontos 2 e 17 (c) e 14 e 17 (d) (c/(c +d)) (Fig. 4). Os percentuais obtidos também foram utilizados na análise de agrupamento com o programa Statistica for Windows, versão 5.1 (STATSOFT 1995). O fenograma foi construído através do método UPGMA, a partir da distância de Manhattan. As configurações de consenso foram computadas a partir das coordenadas originais de cada espécime, através do método de superposição ortogonal pelos quadrados mínimos (M ONTEIRO \& ReIs 1999).

Através dos programas TPSSPLIN versão 1.17 e TPSPLS versão 1.05 (RoHLf 1998), as formas de consenso de cada espécie foram usadas para analisar as deformações relativas, e as figuras obtidas no programa foram usadas para visualizar a direção e magnitude das diferenças na forma. As deformações foram ponderadas com $a=0$, expoente usado para escalar as 

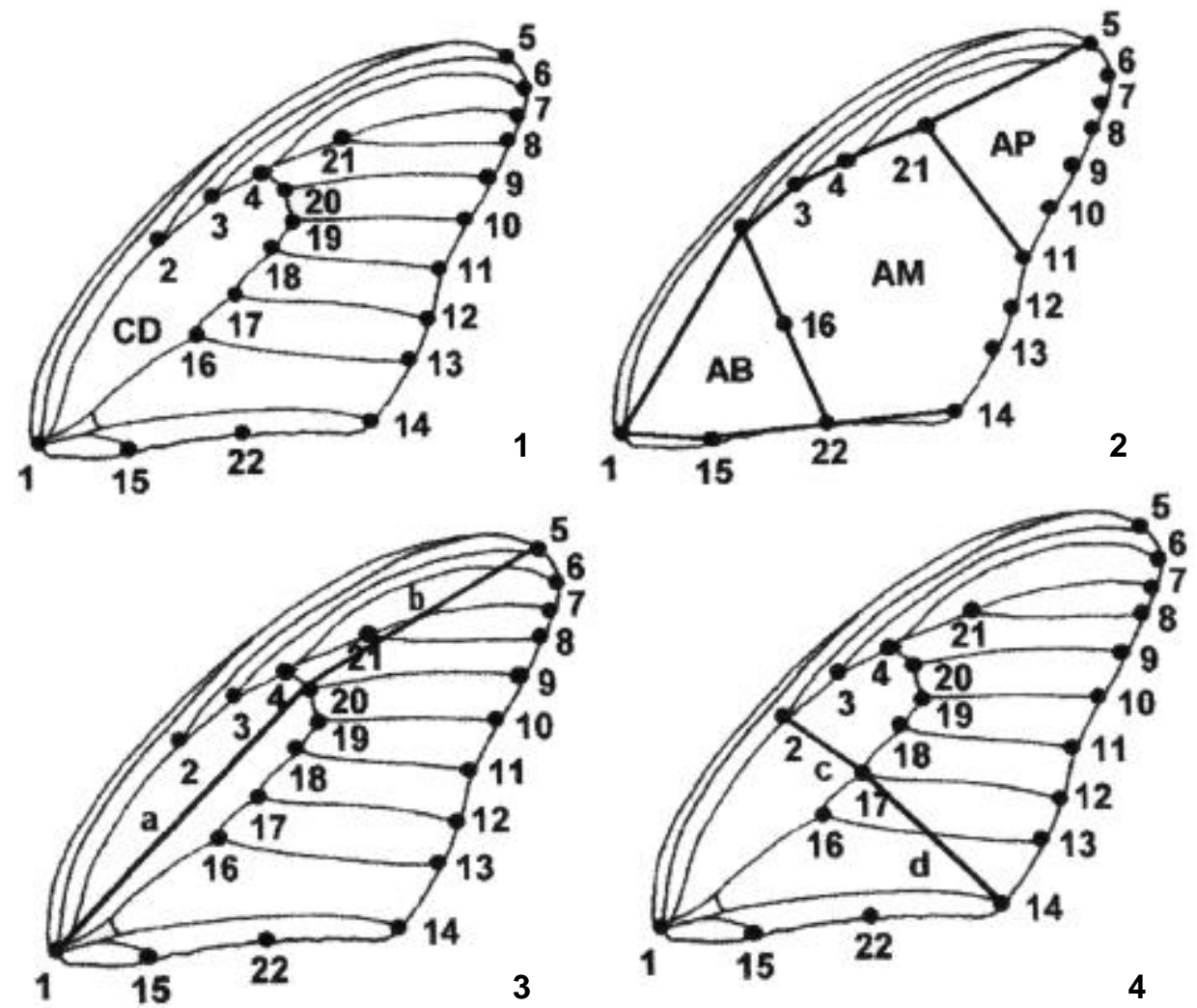

Figuras 1-4. Vista dorsal da asa anterior e 22 marcos anatômicos utilizados nas análises: (1) de coordenadas (CD =célula discal), (2) das regiões alares ( $A B$ = basal; $A M=$ mediana e $A P=$ apical), (3) do comprimento da célula discal (a) e distância até a extremidade apical da asa (b) e (4) da largura da célula discal (c) até a borda da asa (d).

Tabela I. Espécies de Papilioninae examinadas, abreviaturas usadas para referir as espécies, número de borboletas estudadas, por sexo, por espécie e local investigado. (Vdrc) Vale do Diabo + Rincão do Canto, (Rsml) Rincão do Soturno + Morro do Link, (An) Água Negra, (Sccb) Schöenstatt + Camobi.

\begin{tabular}{lcrrrrrr}
\hline \multicolumn{1}{c}{ Espécies } & Abreviaturas & Machos & Fêmeas & Vdrc & Rsml & An & Sccb \\
\hline Mimoides lysithous eupatorion (Lucas, [1859]) & Mile & 15 & 6 & 10 & 7 & 4 & 0 \\
Mimoides lysithous lysithous (Hübner, [1821]) & Mill & 8 & 5 & 7 & 3 & 3 & 0 \\
Mimoides lysithous rurik (Eschscholtz, 1821) & Milr & 9 & 9 & 8 & 9 & 1 & 0 \\
Battus polydamas polydamas (Linnaeus, 1758) & Bapl & 35 & 13 & 4 & 26 & 9 & 9 \\
Battus polystictus polystictus (Butler, 1874) & Bapo & 45 & 11 & 7 & 20 & 27 & 2 \\
Euryades corethrus (Boisduval, 1836) & Euco & 5 & 10 & 0 & 0 & 14 & 1 \\
Parides agavus (Drury, 1782) & Paag & 120 & 31 & 34 & 101 & 16 & 0 \\
Parides anchises nephalion (Godart, 1819) & Paan & 33 & 19 & 14 & 32 & 6 & 0 \\
Parides bunichus perrhebus (Boisduval, 1836) & Pabp & 43 & 10 & 3 & 28 & 22 & 0 \\
Heraclides astyalus astyalus (Godart, 1819) & Heas & 30 & 9 & 8 & 25 & 3 & 3 \\
Heraclides hectorides (Esper, 1794) & Hehe & 19 & 10 & 5 & 17 & 6 & 1 \\
Heraclides thoas brasiliensis (Rothschild \& Jordan, 1906) & Hetb & 11 & 4 & 1 & 3 & 4 & 7 \\
Pterourus scamander scamander (Boisduval, 1836) & Ptsc & 6 & 4 & 0 & 1 & 0 & 9 \\
& Total & 379 & 141 & 101 & 272 & 115 & 32 \\
\hline
\end{tabular}




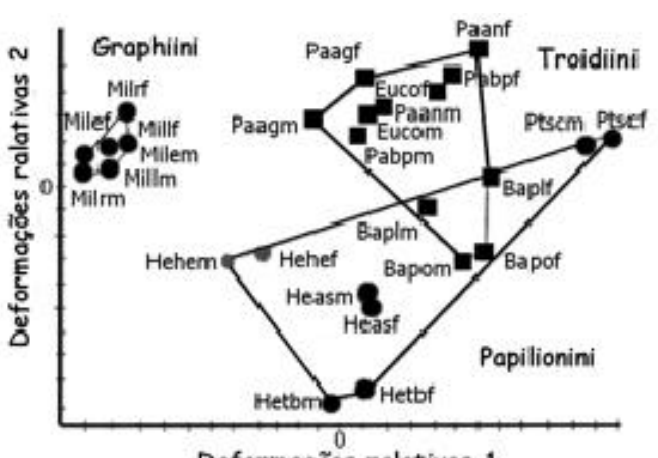

Deformações ralativas 1

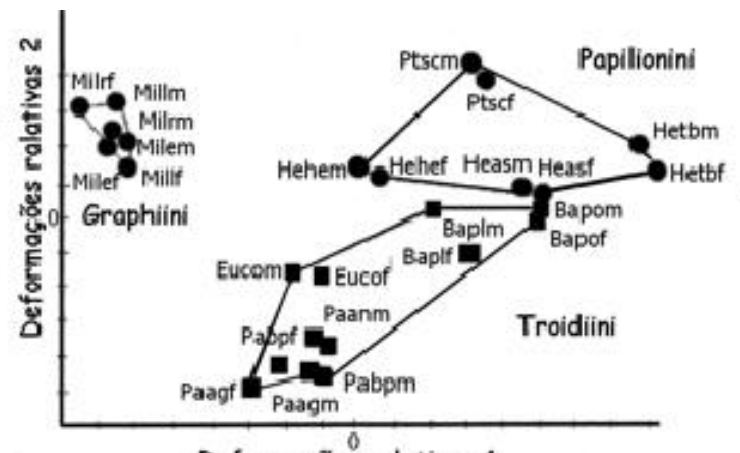

Defarmaçöes ralativas: 1

5

\section{6}

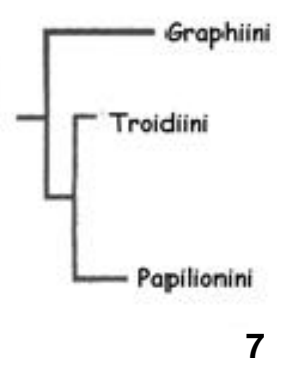

Figuras 5-7. Deformação relativa 1 versus deformação relativa 2, para as formas de consenso de espécies de Papilioninae, por sexo (m = machos, $f=$ fêmeas): (5) com componente uniforme incluído e (6) com componente uniforme excluído, para valores de a =0; (7) Filogenia das tribos (HANCOCK 1983, TYLER et al. 1994).

Tabela II. Valores para as deformações relativas e a proporção explicada da variância, baseadas nas figuras de consenso de machos e fêmeas, das espécies de Papilioninae coletadas nas quatro localidades investigadas. (N) Número de deformações relativas obtidas a partir de 22 marcos anatômicos, quando as espécies foram testadas conjuntamente.

\begin{tabular}{|c|c|c|c|c|}
\hline \multirow{2}{*}{$\mathrm{N}$} & \multicolumn{2}{|c|}{$\alpha=0$ (componente uniforme incluído) } & \multicolumn{2}{|c|}{$\alpha=0$ (componente uniforme excluído) } \\
\hline & Valor único & Proporção da variância (\%) & Valor único & Proporção da variância (\%) \\
\hline 1 & 0,36033 & 55,46 & 0,25209 & 49,44 \\
\hline 2 & 0,19801 & 16,75 & 0,16006 & 19,93 \\
\hline 3 & 0,16025 & 10,97 & 0,11649 & 10,56 \\
\hline 4 & 0,12629 & 6,81 & 0,10583 & 8,71 \\
\hline 5 & 0,10451 & 4,67 & 0,07691 & 4,60 \\
\hline 6 & 0,06751 & 1,95 & 0,05121 & 2,04 \\
\hline 7 & 0,04487 & 0,86 & 0,03931 & 1,20 \\
\hline 8 & 0,04076 & 0,71 & 0,03360 & 0,88 \\
\hline 9 & 0,03566 & 0,54 & 0,03002 & 0,70 \\
\hline 10 & 0,02617 & 0,29 & 0,02458 & 0,47 \\
\hline 11 & 0,02131 & 0,19 & 0,02098 & 0,34 \\
\hline 12 & 0,02031 & 0,18 & 0,01725 & 0,23 \\
\hline 13 & 0,01808 & 0,14 & 0,01581 & 0,19 \\
\hline 14 & 0,01535 & 0,10 & 0,01366 & 0,15 \\
\hline 15 & 0,01446 & 0,09 & 0,01196 & 0,11 \\
\hline 16 & 0,01245 & 0,07 & 0,01073 & 0,09 \\
\hline 17 & 0,01105 & 0,05 & 0,00965 & 0,07 \\
\hline 18 & 0,00959 & 0,04 & 0,00936 & 0,07 \\
\hline 19 & 0,00958 & 0,04 & 0,00805 & 0,05 \\
\hline 20 & 0,00913 & 0,04 & 0,00748 & 0,04 \\
\hline 21 & 0,00758 & 0,02 & 0,00726 & 0,04 \\
\hline 22 & 0,00636 & 0,02 & 0,00625 & 0,03 \\
\hline 23 & 0,00564 & 0,01 & 0,00504 & 0,02 \\
\hline 24 & 0,00505 & 0,01 & 0,00477 & 0,02 \\
\hline 25 & 0,00326 & 0,00 & 0,00287 & 0,01 \\
\hline
\end{tabular}

Revista Brasileira de Zoologia 21(4): 847-855, dezembro 2004 
deformações parciais antes de computar os componentes principais das deformações relativas, de acordo com RohlF (1993, 1996), para estudos sistemáticos exploratórios. Os resíduos da superposição ortogonal pelos quadrados mínimos, das coordenadas originais, para todos os 520 espécimes, foram também submetidos a duas análises separadas de deformações relativas, uma que incluiu o componente uniforme (affine) eoutra que o excluiu. Os programas também foram usados para computar as distâncias de Procrustes, fazendo uso da regressão univariada descrita em Booksteln (1991), onde as coordenadas de cada marco são tratadas como um número complexo com $\mathrm{x}$ correspondendo a parte real e y a imaginária, para analisar as figuras de consenso entre os sexos.

\section{RESULTADOS}

A comparação da covariação das formas de consenso dos machos e das fêmeas, das espécies estudadas, mostra uma correlação alta $(r=0,98)$. Incluindo e excluindo o componente uniforme para $\alpha=0$ (Tab. II), podemos observar claramente que a maior parte da variação morfométrica é devida a soma dos três primeiros valores das deformações relativas observadas. Incluindo o componente uniforme o percentual é de $83,18 \%$ e $79,93 \%$ excluindo o componente uniforme.

O confronto da deformação relativa 1 versus deformação relativa 2 , utilizando as formas de consenso dos machos e das fêmeas de cada espécie, para $\alpha=0$ e excluindo o componente uniforme (Fig. 6), mostra um padrão de classificação similar a filogenia estabel ecida para o grupo (Fig. 7) (HANCOCK 1983, TYLER et al. 1994). Há uma separação clara das espécies no nível de tribo, o que não ocorre com a outra configuração que inclui o componente uniforme (Fig. 5). Analisando a deformação relativa 1, sem o componente uniforme, mostra que Heraclides thoas brasiliensis (Rothschild \& Jordan, 1906) é a espécie que apresenta as asas mais longas e as subespécies de Mimoides as mais curtas. Já a deformação relativa 2 mostra que Pterourus scamander scamander (Boisduval, 1836) tem as asas mais largas e Parides agavus (Drury, 1782), Parides anchises nephalion (Godart, 1819) e Parides bunichus perrhebus (Boisduval, 1836) as mais estreitas. Quando se compara a forma das asas das borboletas capturadas, para as seis espécies com maior número de indivíduos anal isados, através da deformação relativa 1 versus deformação relativa 2, excluindo o componente uniforme, não são observadas evidências que a forma seja diferente entre as quatro localidades investigadas (Fig. 8). Esta observação também pode ser esten dida para as demais espécies e na condição que inclui o componente uniforme.

A figura 9 mostra a análise de agrupamento realizada, utilizando os percentuais relativos da área, do comprimento e da largura da célula discal e das regiões basal, mediana e apical (Figs 1-4, Tab. III). Embora o universo de espécies seja limitado, para uma generalização, o fenograma mostra que os grupos formados (Fig. 4), com esta metodologia, não representam a filogenia das tribos (Fig. 7). Entretanto, a comparação das formas obtidas através de transformação não uniforme (veja Fig. 10) pode explicar al gumas semel hanças observadas na forma das asas entre as espécies. Mimoides lysithous eupatorion (Lucas, [1859]), Mimoides lysithous lysithous (Hübner, [1821]), P. bunichus perrhebus e Heraclides hectorides (Esper, 1794) diferenciam-se das demais espécies por apresentarem células discais com áreas proporcional mente maiores eP. scamander scamander e Euryades corethrus (Boisduval, 1836) proporcionalmente menores. Parides bunichus perrhebus, P. agavus e M. Iysithous mostram células discais mais longas enquanto P. scamander scamander, $\mathrm{H}$. thoas brasiliensis, Heraclides astyalus astyalus (Godart 1819) e Battus polydamas polydamas (Linnaeus, 1758) mais curtas. Heraclides astyalus astyalus, P. scamander scamander e $\mathrm{H}$. hectorides apresentam uma célula discal mais larga e B. polystictus polystictus (Butler, 1874), B. polydamas polydamas e E. corethrus mais estreita. Parides anchises nephalion, $M$. Iysithous lysithous e M. Iysithous rurik (Eschscholtz, 1821) exibem área mediana maior e $\mathrm{H}$. hectorides, $\mathrm{H}$.astyalus astyalus e $\mathrm{H}$. thoas brasiliensis menor área. Heraclides astyalus astyalus eH. hectorides caracterizam-se por apresentarem área basal maior eP. anchises nephalion, $M$. Iysithous rurik, B. polydamas polydamas e $M$.

Tabela III. Proporções das áreas, célula discal, comprimento e largura da célula discal das asas, das espécies de Papilioninae estudadas, com base nas formas obtidas com o componente uniforme excluído. (Mile) Mimoides lysithous eupatorion, (Mill) Mimoides lysithous lysithous, (Milr) M imoides lysithous rurik, (Bapl) Battus polydamas, (Bapo) Battus polystictus, (Euco) Euryades corethrus, (Paag) Parides agavus, (Paan) Parides anchises nephalion, (Pabp) Parides bunichus perrhebus, (Heas) Heraclides astyalus astyalus, (Hehe) Heraclides hectorides, (Hetb) Heraclides thoas brasiliensis, (Ptsc) Pterourus scamander scamander.

\begin{tabular}{|c|c|c|c|c|c|c|c|c|c|c|c|c|c|}
\hline & Mile & Mill & Milr & Bapl & Bapo & Euco & Paag & Paan & Pabp & Heas & Hehe & Hetb & Ptsc \\
\hline Área basal (\%) & 16,94 & 15,31 & 14,96 & 15.29 & 15.91 & 16.94 & 18,01 & 14,14 & 17,40 & 19,30 & 20,54 & 17,95 & 15,67 \\
\hline Área mediana (\%) & 63,93 & 66,60 & 66,53 & 62,45 & 61,21 & 64,91 & 60,10 & 68,31 & 63,61 & 56,73 & 55,17 & 55,35 & 61,49 \\
\hline Área apical (\%) & 19,14 & 18,09 & 18,51 & 22,26 & 22,88 & 18,15 & 21,89 & 17,55 & 18,99 & 23,96 & 24,29 & 26,70 & 22,83 \\
\hline Área discal (\%) & 21,57 & 21,18 & 19,38 & 18,76 & 18,46 & 17,75 & 20,25 & 18,46 & 20,49 & 19,48 & 20,06 & 18,79 & 16,84 \\
\hline $\begin{array}{l}\text { Comprimento da } \\
\text { célula discal (\%) }\end{array}$ & 55,86 & 59,29 & 55,74 & 55,38 & 55,55 & 56,34 & 59,63 & 55,51 & 60,01 & 52,19 & 56,46 & 51,30 & 47,66 \\
\hline $\begin{array}{l}\text { Largura da célula } \\
\text { discal (\%) }\end{array}$ & 31,43 & 29,80 & 28,63 & 28,38 & 27,29 & 29,68 & 32,32 & 28,57 & 30,05 & 34,90 & 30,19 & 31,90 & 30,92 \\
\hline
\end{tabular}


Battus p. polydamas

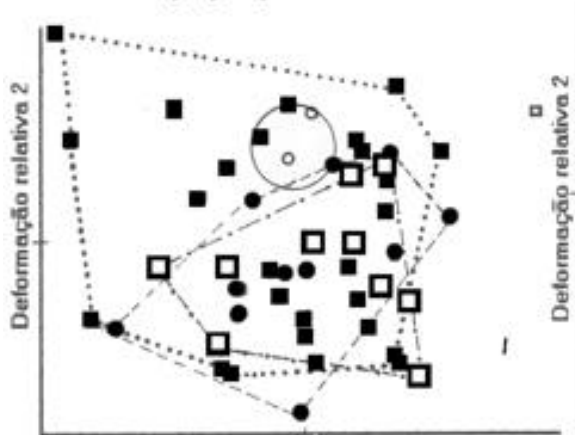

Battus p. polystictus
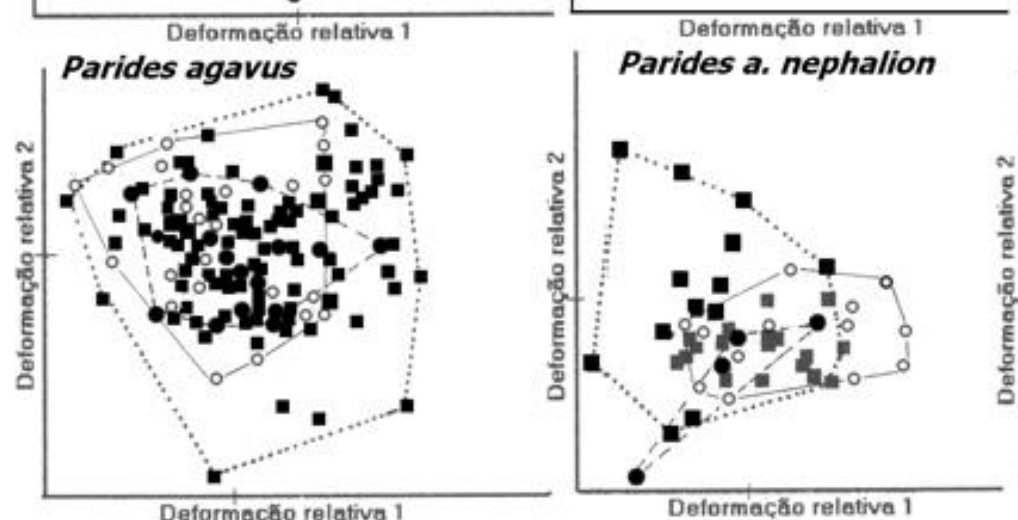

Deformaçẵo reletiva 1

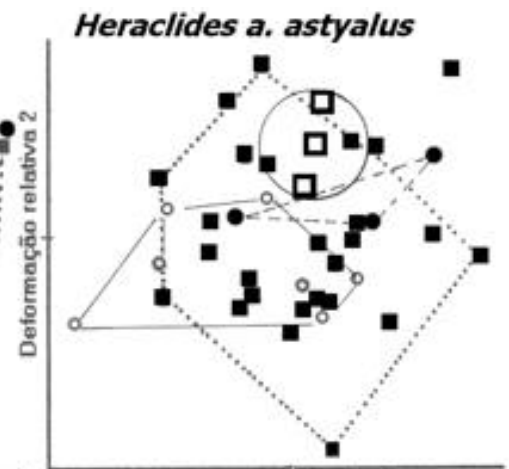

Deformaçăo relative 1

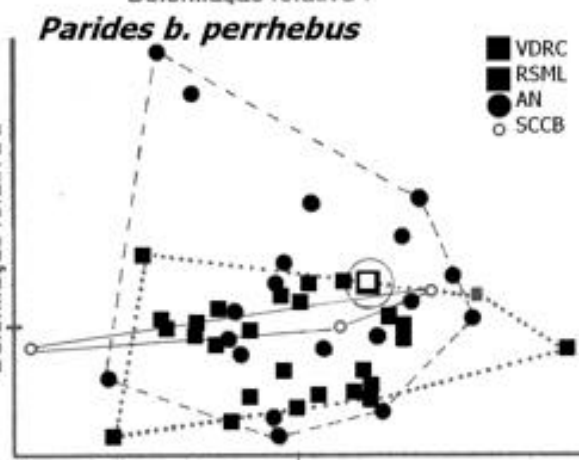

Daformaçắo relativa 1

Figura 8. Deformações relativas 1 versus deformações relativas 2, para as seis espécies de Papilioninae, com maior número de borboletas analisadas, levando em conta o local da captura, com o componente uniforme excluído.

Iysithous lysithous a menor. Heraclides astyalus astyalus, $\mathrm{H}$. thoas brasiliensis eH. hectorides exibem a maior área apical eP. anchises nephalion, M. Iysithous lysithous, E. corethrus e M. Iysithous rurik a menor

\section{DISCUSSÃ O}

A análise quantitativa de marcos anatômicos tem fornecido evidên cias sobre a variação na simetria das formas e sobre a variabilidade inerente, que pode ser biologicamente relevante (Daly 1985, Rohlf 1990, Strauss 1990).

O al to grau de correlação estimado neste estudo indica que a forma da asa anterior não é útil para discriminar os sexos, pois as formas médias de machos e fêmeas não diferem nem no comprimento e nem na largura.

A análise das deformações relativas mostra que as diferenças na forma das asas das espécies estudadas não podem ser explicadas por fenômenos que modificam a forma como um todo. Sugere-se, ainda, que diferentes regiões da asa podem desenvolver sua forma independentemente uma das outras (M onteiro \& ReIs 1999). Tal observação pode ser utilizada futuramente para identificar padrões de variação ou para analisar novas maneiras de descrever e avaliar alterações na forma da asa destas borbol etas. O dimorfismo sexual com base na forma da asa não pode ser utilizado como um caractere discrimi-

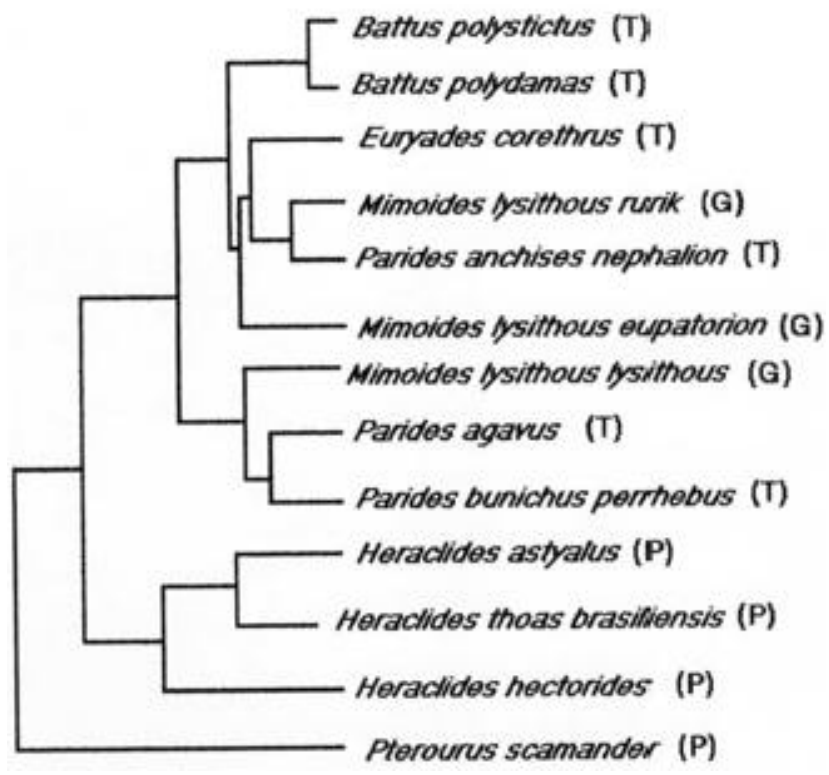

Figura 9. Fenograma das espécies de Papilioninae estudadas, com base nos percentuais das áreas das asas (basal, mediana e apical) e da área, do comprimento e da largura da célula discal. As tribos estão indicadas entre parênteses: Graphiini (G), Papilionini (P) eTroidini (T).

Revista Brasileira de Zoologia 21(4): 847-855, dezembro 2004 

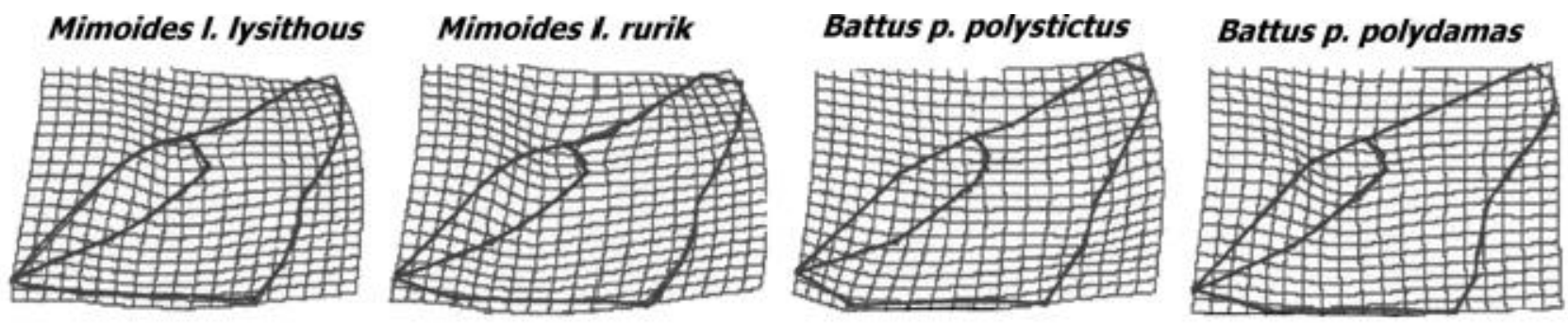

\section{Euryades corethrus}
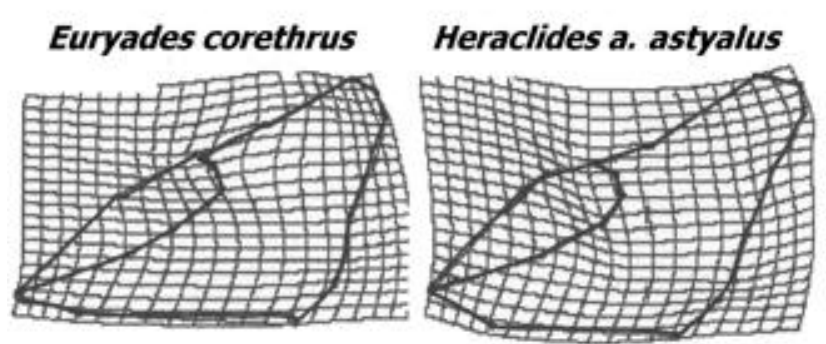
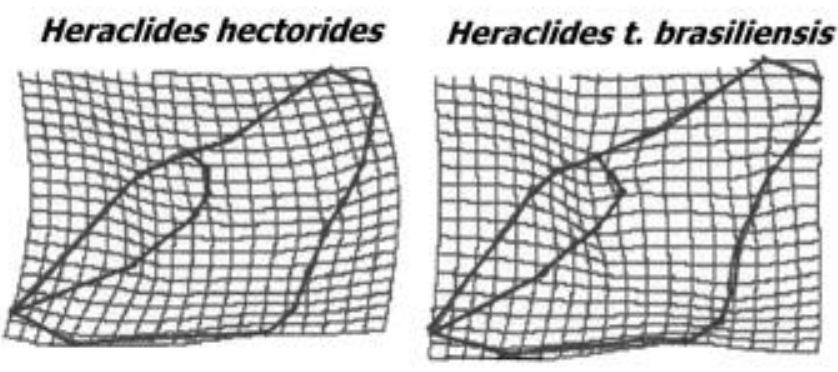

Parides agavus

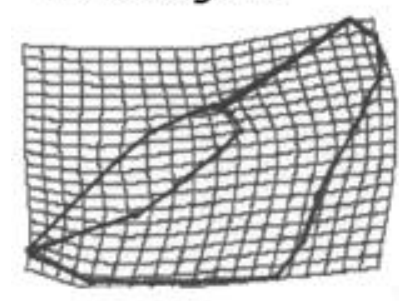

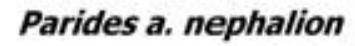

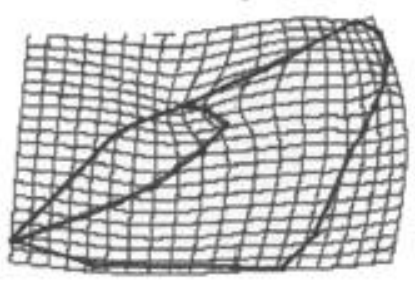

Parides b. perrhebus

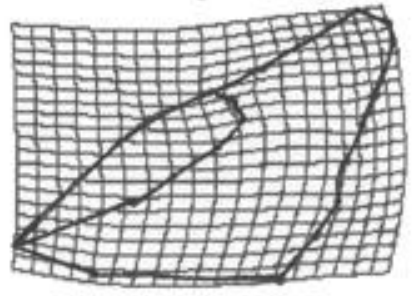

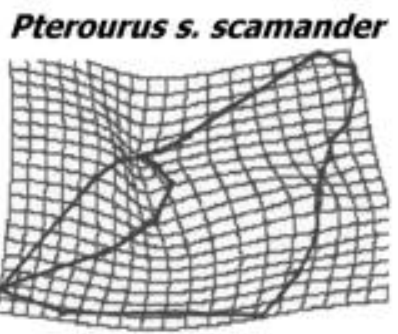

Figura 10. Variação na forma da asa anterior de 11 espécies de Papilioninae, utilizando a função "thin plate splines", com componente uniforme removido.

natório, já que as formas de consenso de machos e fêmeas, de uma mesma espécie, ocupam posições próximas, quando confrontadas as deformações relativas (Figs 5-7). Deste modo, com base nos gêneros analisados, diferenças na forma da asa, entre os sexos de Papilioninae, não podem ser determinadas através de morfometria geométrica. Entretanto, tal observação pode ser útil em estudos filogenéticos, quando o número de borboletas analisadas em um dos sexos é pequeno em relação ao outro. A falta de distinção na forma da asa entre as local idades estudadas denota que a forma é conservativa e deve estar sob forte sel eção estabilizadora, não permitindo variações que se afastem muito da forma mais freqüente. Por outro lado, a semelhança observada entre as local idades investigadas demonstra a necessidade de estudar e determinar os limites geográficos que podem produzir diferenciações locais. As semel hanças e recorrências entre os valores percentuais denotam que os grupamentos formados podem revelar padrões evolutivos associados ao comportamen- to ecológico das espécies. Estudos que agregassem a forma da asa com ambientes e comportamentos ecológicos poderiam explicar a similaridade observada na estrutura e na forma da asa, como por exemplo, mecanismos associados com o vôo e deslocamento na área de vida, ou entre o vôo e comportamento de escape em relação a predadores (DUDLEY 2000).

Os resultados obtidos neste estudo podem ser entendidos como uma contribuição ao estudo de marcos anatômicose a aspectos na variação da forma da asa anterior de Papilioninae. Estes resultados também exemplificam como o método pode enriquecer a nossa avaliação na descrição da forma. Contudo, o estudo de marcos anatômicos deve ser compreendido como um procedimento auxiliar na descrição e não como protocolo para substituir a descrição destas borboletas. Todavia, esta prudência não deve impedir que os resultados possam ser analisados com discernimento e confiança nas diferenças naturais da forma entre organismos (NAYLOR 1996). 


\section{A GRA DECIMENTOS}

À CAPES pela bolsa PICD. Ao Dr. Roberto E. Reis (PUCRS), pela leitura crítica do trabalho.

\section{REFERÊNCIAS BIBLIOGRÁ FICAS}

AlexAnder, R. McN. 1983. Animal Mechanics. Seattle, University of Washington, $X+346 p$.

BARBAult, R. 1988. Body size, ecological constraints, and the evolution of life-history strategies, p. 261-286. In: M.K. Hecht; B. Wallace \& G.T. Prance (Eds). Evolutionary Biology. Plenum, New York.

BooKSTEIN, F.L. 1991. Morphometric tools for landmark data: Geometry and Biology. Cambridge, Cambridge University Press, XVII+455p.

BRaKefield, P. 1979. Spot number in Maniola jurtina - variation between generations and selection in marginal populations. Heredity, London, 42: 259-266.

Brown JR., K.S.; A.J. Damman \& P. Feeny. 1981. TroidineSwallwtails (Lepidoptera: Papilionidae) in Southeastern Brazil: natural history and foodplant relationships. Journal of Research on the Lepidoptera, Beverly Hills, 19 (4): 199-226.

CoRKUM, L.D. 1987. Patterns in mayfly (Ephemeroptera) wing length: Adaptation to dispersal? The Canadian Entomologist, Ottawa, 119: 783-790.

D'AlmeidA, R.F. 1966. Catálogo dos Papilionidae Americanos. São Paulo, Sociedade Brasileira de Entomologia, VI+366p.

DALY, H.V. 1985. Insect morphometrics. Annual Review of Entomology, Palo Alto, 30: 415-438.

DudLEY, R. 2000. The biomechanics of insect flight. Princeton, Princeton University Press, XII+476p.

HANCOCK, D.L. 1983. Classification of the Papilionidae (Lepidoptera): a phylogenetic approach. Smithersia, Bulawayo, 2: 1-48.

HARDER, L.D. 1985. Morphology as a predictor of flower choice by Bumble Bees. Ecology, Washington, 66 (1): 198- 210.

Kingsolver, J.G. \& D.C. Wiernasz. 1987. Dissecting correlated characters: Adaptive aspects of phenotypic covariation in melanization pattern of Pieris butterflies. Evolution, Philadel phia, 41: 491-503.

KLeIn, R.M. 1983. Aspectos fitofisionômicos da floresta estacional na fralda da Serra Geral (RS). In: Anais do Congresso Nacional de Botânica, Porto Alegre, 192p.

MARCUS, L.F. 1990. Traditional morphometrics, p. 77-120. In: F.J. Rohlf \& F.C. Bookstein (Eds). Proceedings of the Michigan morphometrics workshop. Ann Arbor, TheUniversity of Michigan, Museum of Zoology, Special publication number 2, 380p.

MARCUS, L.F. 1993. Some aspects of multivariate statistics for morphometrics, p, 95-130. In: L.F. Marcus; E. Bello \& A. GARCIA-VALDECASAS (Eds). Contributions to Morphometrics. Madrid, Museo Nacional de Ciencias Naturales (CSIC), vol. 8, 264p.
MASON, L.G.; P.R. EhrLich \& T.C. Emmel. 1968. The population biology of butterfly Euphydryas editha. VI. Phenetics of the Jasper Ridge colony 1965-1966. Evolution, Philadelphia, 22: 46-54.

Monteiro, L.R. \& S.F. Reis. 1999. Princípios de Morfometria Geométrica. Ribeirão Preto, Holos Editora Ltda, $X+188 p$.

Morais, A.B.B. DE; K.S. BRown JR. 1992. Larval foodplant and other effects on Troidine guild composition (Papilionidae) in southeastern Brazil. Journal of Research on the Lepidoptera, Beverly Hills, 30 (1-2): 19-37.

NACHTIGALL, W. 1967. Aerodinamiche Messungen am Tradflugelsystem segelner Schmetterlinge. Zeitschrift für Vergleichende Physiologie, Berlin, 54: 210-231.

NAYLOR, G.J.P. 1996. Introduction to comparisons among taxa at the level of species. genus and family, p. 437-439. In: L. Marcus; M. Corti; A. Loy; G. Naylor; D.E. Slice (Eds). Advances in Morphometrics. New York, Plenum Publishing, NATO ASI Series A: Life Sciences, vol. 284, 587p.

Pearse, F.K. \& N.D. Murray. 1982. Sex and variability in the common brown butterfly Heteronympha merope merope (Lepidoptera: Satyrinae). Evolution, Philadelphia, 36: 1251-1264.

Rohlf, F.J. 1990. Morphometrics. Annual Review of Ecology and. Systematics, Palo Alto, 21: 299-316.

. 1993. Relative warp analysis and an example of its application to mosquito wings, p. 131-159. In: L.F. M ARCUS; E. Bello \& A. Garcia-Valdecasas (Eds). Contributions to morphometrics. Madrid, Museo Nacional de Ciencias Naturales (CSIC), vol. 8, 264p.

. 1996. Morphometric spaces. Shape components and the effect of linear transformations, p. 117-130. In: L. M ARCUS; M. Corti; A. Loy; G. Naylor; D.E. Suice (Eds). Advances in Morphometrics. New York, Plenum Publishing, NATO ASI Series A: Life Sciences, vol. 284, 587p.

. 1998. TPSrel $w$ for Windows version 1.17. Thin Plate Spline Relative Warps Analysis. Department of Ecology and Evolution, State University of New York, Stony Book. On line at: http://life.bio.sunysb.edu/morph/index.html. Acesso em: $28 / X I / 2002$.

. 2001. TPSDIG32 for Windows version 1.18. Department of Ecology and Evolution, State University of New York, Stony Book. On line at: http://life.bio.sunysb.edu/ morph/index.html. Acesso em: 28/XI2002.

Schwartz, G. \& R.A. Dı Mare. 2001. A diversidade de quinze espécies de borboletas (Lepidoptera: Papilionidae) em sete comunidades de Santa Maria, RS. Ciência Rural, Santa Maria, 31 (1): 49-55.

Strauss, R.E. 1990. Patterns of quantitative variation in lepidopteran wing morphology: the convergent groups Heliconiinae and Ithomiinae (Papilionoidea: Nymphalidae). Evolution, Philadelphia, 44 (1): 86-103.

STATSOF. 1995. Statistica for Windows (Computer program manual). Tulsa, OK, Stat Soft.

TESTON, J.A. \& E. CorseuIL. 1998. Lista documentada dos papilio-

Revista Brasileira de Zoologia 21(4): 847-855, dezembro 2004 
nídeos (Lepidoptera, Papilionidae) do Rio Grande do Sul, Brasil. Biociências, Porto Alegre, 6: 81-94.

TYLER, H.A.; K.S. BRown JR. \& K.H. WILSON. 1994. Swallowtail butterflies of the Americas: a study in biological dynamics, ecological diversity, biosystematics and conservation.
Gainesville, Scientific Publishers, 376p.

WILCOX, D.; B. DoWE; D. McDAVID \& D. Greer.1996. Image Tool Windows 98. Microsoft Corp., Image Tool version 2.0 al pha 3, developed at the University of Texas Health ScienceCenter at San Antonio, Texas.

Recebido em 02.III.2004; aceito em 04.XI.2004. 